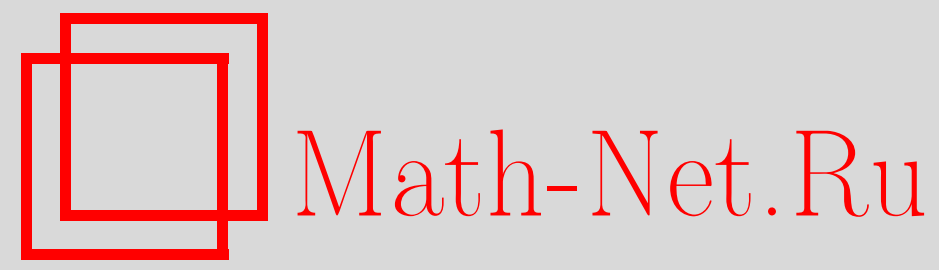

В. И. Пагурова, Об асимптотической мощности критерия обнаружения выбросов, Теория вероятн. и ее примен., 1997, том 42, выпуск 3, 482-495

DOI: https://doi.org/10.4213/tvp1947

Использование Общероссийского математического портала Math-Net.Ru подразумевает, что вы прочитали и согласны с пользовательским соглашением http://www . mathnet.ru/rus/agreement

Параметры загрузки:

IP : 52.6 .47 .48

26 апреля 2023 г., 13:36:35

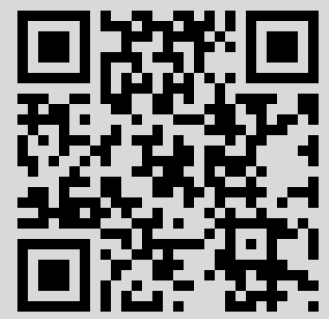




\section{ОБ АСИМПТОТИЧЕСКОЙ МОЩНОСТИ КРИТЕРИЯ ОБНАРУЖЕНИЯ ВЫБРОСОВ}

Для двупараметрического семейства распределений, зависящего от неизвестных параметров сдвига и масштаба, исследуется асимптотическая мощность критерия обнаружения выбросов, впервые предложенного в работе [1]. Среди других полезных характеристик критерия исследуется вероятность ошибочного принятия «хорошего» наблюдения в качестве выброса и вероятность обнаружения «плохого» наблюдения в качестве выброса. Для устранения маскирующего эффекта предлагается использовать робастные оценки параметров сдвига и масштаба, имеюшие высокую пороговую точку и ограниченную функцию влияния, в терминологии Хампеля [8], [9]. Отметим, что в работах [4], [6] исследуется скорость сходимости нормированного максимума независимых одинаково распределенных случайных величин для однопараметрических семейств распределений, зависящих от неизвестного параметра сдвига. Работа [5] посвящена исследованию асимптотических свойств критерия обнаружения выбросов для эллиптического семейства многомерных распределений.

Ключевые слова и фразы: критерий для выбросов, асимптотическая мошность, двупараметрическое семейство распределений.

1. Построение статистической процедуры. Пусть $X_{1}$, $X_{2}, \ldots, X_{n}$ независимы, $X_{i}$ имеет непрерывную функцию распределения (ф.p.) $F\left(\left(x-\theta_{1 i}\right) / \theta_{2}\right), i=1, \ldots, n$. Рассмотрим гипотезу $H_{0}: \theta_{11}=$ $\theta_{12}=\cdots=\theta_{1 n}$, против альтернативы $H_{1}$ : среди $X_{1}, \ldots, X_{n}$ имеются наблюдения, для которых $\theta_{1 i}>\theta_{1}$, для остальных наблюдений имеем $\theta_{1 i}=\theta_{1}$; число наблюдений, для которых $\theta_{1 i}>\theta_{1}$, не более $k$. Параметры $\left(\theta_{11}, \ldots, \theta_{1 n}, \theta_{1}, \theta_{2}\right)$ неизвестны, число $k \geqslant 1$ задано.

Наблюдение $X_{i}$, для которого $\theta_{1 i}>\theta_{1}$, будем называть наблюдением, засоряющим выборку (н.з.в.).

Предположим, что при гипотезах $H_{0}$ и $H_{1}$ и фиксированном $k$ существуют $n^{\varepsilon}$ - и $n^{\delta}$-состоятельные оценки $\hat{\theta}_{1}$ и $\hat{\theta}_{2}$ для $\theta_{1}$ и $\theta_{2}$ соответственно, построенные на основе данных $X_{1}, \ldots, X_{n}$ (будем считать, что оценка $T_{n}(X)$ является $n^{\varepsilon}$-состоятельной оценкой для параметра $\theta$, если

*Московский государственный университет им. М. В. Ломоносова, Воробъевы Горы, 119899 Москва, Россия.

1) Работа выполнена при поддержке Российского фонда фундаментальных исследований, грант 96-01-01919. 
$n^{\varepsilon}\left(T_{n}(X)-\theta\right)$ ограничена по вероятности при $\left.n \rightarrow \infty\right)$. В качестве таких оценок следует использовать робастные ощенки, сохраняющие свойство состоятельности в присутствии наблюдений, засоряющих выборку. Асимптотические свойства робастных оценок исследуются в монография्रX [8], [9].

Введем величины

$$
Y_{i}=\frac{X_{i}-\theta_{1}}{\theta_{2}}, \quad Z_{i}=\frac{X_{i}-\hat{\theta}_{1}}{\hat{\theta}_{2}},
$$

$i=1, \ldots, n, Y_{(1)} \leqslant Y_{(2)} \leqslant \cdots \leqslant Y_{(n)}$ и $Z_{(1)} \leqslant Z_{(2)} \leqslant \cdots \leqslant Z_{(n)}$ означают соответствуюшие вариационные ряды.

Пусть $\Delta=\min (\varepsilon, \delta), y=y_{n}(t)$ означает корень уравнения

$$
F(y)=1-\frac{t}{n}
$$

Лемма 1.1. Если для любого фиксированного $x>0$

$$
\begin{aligned}
& \lim _{n \rightarrow \infty} n\left[1-F\left(y \pm \frac{y}{x n^{\Delta}}\right)\right]=t, \\
& \lim _{n \rightarrow \infty} n\left[1-F\left(y \pm \frac{1}{x n^{\Delta}}\right)\right]=t,
\end{aligned}
$$

тогда при справедливости гипотезы $H_{0}$, яюбом иелом фиксированном $m \geqslant 0 u n \rightarrow \infty$

$$
\frac{Z_{(n-m)}-y}{Y_{(n-m)}-y} \stackrel{\mathbf{P}}{\longrightarrow} 1 .
$$

Доказательство леммы 1.1 и всех последующих утверждений помещено в приложении.

Следствие 1.1. Если при некоторых $a_{n}>0$ и $b_{n}$ величина $\left(Y_{(n)}\right.$ $\left.b_{n}\right) / a_{n}$ при $n \rightarrow \infty$ имеет предельное невырожденное распределение, то-

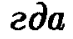

1) условие (2) эквивалентно условию

$$
\lim _{n \rightarrow \infty}\left[\frac{b_{n}}{a_{n} n^{\Delta}}\right]=0 ;
$$

2) условие (3) эквивалентно условию

$$
\lim _{n \rightarrow \infty}\left(a_{n} n^{\Delta}\right)=\infty
$$

Обозначим $p_{i i}=0, i \geqslant 1, p_{10}=\exp (-t)$, введем величины $p_{k j} \mathrm{c}$ помошью рекуррентного соотношения $p_{k j}=\exp (-t) \sum_{i=0}^{j} p_{k-1, i} t^{j-i} /(j-$ $i) !, j=0,1, \ldots, k-1$, величину $t$ найдем из условия

$$
\sum_{j=0}^{k-1} p_{k j}=1-\alpha
$$


Теорема 1.1. Если выполняются условия (2) $u(3), y_{n i}=y_{n}(t i)$, $i=1, \ldots, k$, определяются из уравнения (1), а $t$ удовлетворяет coотношению (7), то при справедливости аипотезы $H_{0}$

$$
\lim _{n \rightarrow \infty} \mathbf{P}\left\{Z_{(n)}<y_{n 1}, Z_{(n-1)}<y_{n 2}, \ldots, Z_{(n-k+1)}<y_{n k}\right\}=1-\alpha .
$$

Теорема 1.1 позволяет построить следующую статистическую процедуру проверки гипотезы $H_{0}$ против $H_{1}$.

Зададим уровень значимости $\alpha$ и рассмотрим вектор $\left(Z_{(n)}\right.$, $\left.Z_{(n-1)}, \ldots, Z_{(n-k+1)}\right)$. Если событие $\left\{Z_{(n-j+1)} \geqslant y_{n j}\right\}$ имеет место хотя бы для одного $j \subseteq\{1,2, \ldots, k\}$, то гипотеза $H_{0}$ отвергается в пользу альтернативы $H_{1}$, при этом все значения $j \subseteq\{1,2, \ldots, k\}$, для которых выполняется событие $\left\{Z_{(n-j+1)} \geqslant y_{n j}\right\}$, определяют выбросы $X_{(n-j+1)}$. В предположениях теоремы 1.1 при $n \rightarrow \infty$ уровень значимости критерия стремится к $\alpha$.

2. Асимптотические свойства оценок при альтернативе. В дальнейшем будем считать, что $k=2$. Случай $k>2$ рассматривается аналогичным образом. Введем события

$$
\begin{aligned}
D_{0}=\left\{Z_{(n)}<y_{n 1}, Z_{(n-1)}<y_{n 2}\right\}, & D_{11}=\left\{Z_{(n)} \geqslant y_{n 1}, Z_{(n-1)}<y_{n 2}\right\}, \\
D_{12}=\left\{Z_{(n)}<y_{n 1}, Z_{(n-1)} \geqslant y_{n 2}\right\}, & D_{13}=\left\{Z_{(n)} \geqslant y_{n 1}, Z_{(n-1)} \geqslant y_{n 2}\right\},
\end{aligned}
$$

$D_{1}=\bar{D}_{0}=D_{11} \cup D_{12} \cup D_{13}$. Всюду далее $\mathbf{P} \mathbf{c}$ индексом 0 или 1 означает вероятность события в условиях справедливости соответствуюшей гипотезы; например, $\mathbf{P}_{0}\{A\}$ означает вероятность события $A$ при гипотезе $H_{0}$.

Альтернативу $H_{1}$ представим в виде $H_{1}=H_{11} \cup H_{12}$, где

$$
\begin{aligned}
& H_{1 j}=\left\{\text { среди } X_{1}, \ldots, X_{n} \text { имеется ровно } j\right. \text { наблюдений с } \\
& \\
& \theta_{1 i}=\theta_{2}\left(y_{n 1}+\tau\right)+\theta_{1}, \text { остальные наблюдения } \\
&\text { имеют } \left.\theta_{1 i}=\theta_{1}\right\}, \quad j=1,2 .
\end{aligned}
$$

Постоянную $\tau$ найдем из того условия, что предел вероятности ошибки второго рода не превосходит $\beta, \lim _{n \rightarrow \infty} \mathbf{P}_{1}\left\{D_{0}\right\} \leqslant \beta$. Выбор альтернативы такого рода диктуется тем, что существует нетривиальный предел $\lim _{n \rightarrow \infty} \mathbf{P}_{1}\left\{D_{0}\right\}$.

Далее мы рассмотрим пример ощенки параметров $\left(\theta_{1}, \theta_{2}\right)$, являющейся $\sqrt{n}$-состоятельной как при гипотезе $H_{0}$, так и при альтернативе $H_{1}=H_{11} \cup H_{12}$. Для этого нам понадобится следующая

Лемма 2.1. Если $f(x)=F^{\prime}(x)$ непрерывно дифференчируема в окрестностях точек $\zeta_{i}=F^{-1}\left(\lambda_{i}\right), f\left(\zeta_{i}\right) \neq 0, n_{i}=\left[n \lambda_{i}\right]+1, i=1,2$, $0<\lambda_{1}<\lambda_{2}<1$, то при гипотезе $H_{1}=H_{11} \cup H_{12}$ совместное асимптотическое распределение вектора

$$
\left(\sqrt{n}\left(Y_{\left(n_{1}\right)}-\zeta_{1}\right), \sqrt{n}\left(Y_{\left(n_{2}\right)}-\zeta_{2}\right)\right)
$$


при $n \rightarrow \infty$ является двумерным нормальным распределением с вектором нулевых математических ожиданий и ковариачионной матричеน̆

$$
\Lambda=\left(\begin{array}{cc}
\frac{\lambda_{1}\left(1-\lambda_{1}\right)}{f^{2}\left(\zeta_{1}\right)} & \frac{\lambda_{1}\left(1-\lambda_{2}\right)}{f\left(\zeta_{1}\right) f\left(\zeta_{2}\right)} \\
\frac{\lambda_{1}\left(1-\lambda_{2}\right)}{f\left(\zeta_{1}\right) f\left(\zeta_{2}\right)} & \frac{\lambda_{2}\left(1-\lambda_{2}\right)}{f^{2}\left(\zeta_{2}\right)}
\end{array}\right) .
$$

В условиях леммы 2.1 для симметричных распределений выборочная медиана $\operatorname{med}_{1 \leqslant i \leqslant n} X_{i}=X_{([n / 2]+1)}$ и абсолютное медианное отклонение

$$
S_{n}=1.49 \underset{1 \leqslant i \leqslant n}{\operatorname{med}}\left|X_{i}-\underset{1 \leqslant j \leqslant n}{\operatorname{med}} X_{j}\right|
$$

(симметризованная версия выборочного интерквартильного размаха $\left(X_{([3 n / 4])}-X_{([n / 4]+1)}\right) /\left(F^{-1}(0.75)-F^{-1}(0.25)\right)$, в которой коэффициент 1.49 обеспечивает состоятельность оценки в случае нормального распределения) являются $\sqrt{n}$-состоятельными оценками $\theta_{1}$ и $\theta_{2}$ соответственно как при гилотезе $H_{0}$, так и при альтернативе $H_{1}=H_{11} \cup H_{12}$. Кроме того, векторная ощенка $\left(\operatorname{med}_{1 \leqslant i \leqslant n} X_{i}, S_{n}\right)$ является примером робастной оцепки для $\left(\theta_{1}, \theta_{2}\right)$, устойчивой к присутствию в выборке н.з.в., имеющей высокую пороговую точку и ограниченную функцию влияния [8], [9].

Обозначим (А) класс распределений $F(x)$, для которых при $x \rightarrow \infty$

$$
1-F(x) \sim a x^{c} \exp \left(-b x^{\gamma}\right), \quad a, b, \gamma>0, \quad|c|<\infty
$$

символом (В) обозначим класс распределений $F(x)$, для которых при $x \rightarrow \infty$

$$
1-F(x) \sim a x^{-\gamma}, \quad a, \gamma>0 .
$$

Тогда для класса (A) при $n \rightarrow \infty$ имеем

$$
y_{n}(t) \sim\left(\frac{1}{b} \ln \frac{n}{t}\right)^{1 / \gamma}
$$

для класса (В) при $n \rightarrow \infty$ имеем

$$
y_{n}(t) \sim\left(\frac{a n}{t}\right)^{1 / \gamma}
$$

Заметим, что при $\boldsymbol{n} \rightarrow \infty$ для класса (A) выполняются соотношения $b_{n} / a_{n}=O(\ln n)$ и $a_{n}=O\left((\ln n)^{(1-\gamma) / \gamma}\right)$, для класса (В) выполняются условия $b_{n}=0$ и $a_{n}=O\left(n^{1 / \gamma}\right)$ (см., например, [2]), так что условия (5) и (6) выполняются как для класса (А), так и для класса (В).

В дальнейшем мы будем исследовать предельное при $n \rightarrow \infty$ поведение вероятности $\mathbf{P}_{1}\left\{D_{1}\right\}$, используя ощенки $\hat{\theta}_{1}$ и $\hat{\theta}_{2}$, сохраняющие свойства состоятельности при альтернативе $H_{1}=H_{11} \cup H_{12}$. Для проведения этого исслецования нам потребуется следующая 
Лемма 2.2. Пусть величина у определяется соотночением (1), $\hat{\theta}_{1} \quad u \hat{\theta}_{2}$ означают $n^{\varepsilon}-u n^{\delta}$-состоятельные оченки $\theta_{1}$ u $\theta_{2}$ соответственно при альтернативе $H_{1}=H_{11} \cup H_{12}$, тогда при альтернативе $H_{1}=H_{11} \cup H_{12}$ для распределений из класса (В) при условии $\Delta \gamma>1 u$ для распределений из класса (A) при любом челом биксированном $m \geqslant 0$ $u n \rightarrow \infty$ имеем

$$
\frac{Z_{(n-m)}-y}{Y_{(n-m)}-y} \stackrel{\mathbf{P}}{\longrightarrow} 1 \text {. }
$$

3. Меры, характеризуюшие качество критерия. При $k=2$ в предположениях теоремы 1.1 вероятность $\mathbf{P}_{0}\left\{D_{0}\right\} \rightarrow \exp (-2 t)(1+t) n \rightarrow$ $\infty$. Следовательно, величина $t$ является решением уравнения $\exp (-2 t)(1+t)=1-\alpha$. Таким образом, при $\alpha \rightarrow 0$ имеем $t=\alpha+O\left(\alpha^{3}\right)$.

Наблюдение, засоряющее выборку, обозначим $X_{c}$. Введем события

$$
\begin{gathered}
E_{i}=\left\{\text { существуют ровно } i \text { н.3.в., } X_{c_{j}}=X_{(n-j+1)}, j=1, i\right\} \\
i=1,2, \quad E=E_{1} \cup E_{2} .
\end{gathered}
$$

Нас будет интересовать предельное при $n \rightarrow \infty$ значение мощности

$$
I_{1}=\lim \mathbf{P}_{1}\left\{D_{1}\right\}
$$

при альтернативах, указанных в п. 2, а также предельное значение $I_{2}$ вероятности того, что критерий ошибочно признает «хорошее» наблюдение в качестве н.з.в., т.е. значение

$$
I_{2}=\lim \mathbf{P}_{1}\left\{D_{1}\right\}-\lim \mathbf{P}_{1}\left\{D_{1} E\right\}
$$

Обозначим

$$
I_{3}=\lim \mathbf{P}_{1}\left\{D_{1} \mid E\right\}, \quad I_{4}=\lim \mathbf{P}_{1}\{E\}
$$

- предельное значение вероятности того, что н.з.в. обнаруживается как выброс. Следуя монографии [7], будем считать, что всякий разумный критерий полжен иметь большие значения величин $I_{1}, I_{3}, I_{4}$ и малое значение $I_{2}$.

Представляют интерес также частные меры

$$
\begin{array}{lll}
I_{5}=\lim \mathbf{P}_{12}\left\{D_{11} \mid E\right\}, & I_{6}=\lim \mathbf{P}_{11}\left\{D_{11} \mid E\right\}, & I_{7}=\lim \mathbf{P}_{12}\left\{D_{12} \mid E\right\} \\
I_{8}=\lim \mathbf{P}_{11}\left\{D_{12} \mid E\right\}, & I_{9}=\lim \mathbf{P}_{12}\left\{D_{13} \mid E\right\}, & I_{10}=\lim \mathbf{P}_{11}\left\{D_{13} \mid E\right\}
\end{array}
$$

В дальнейшем мы разделим все распределения из классов (A) и (B) на три подгруппы. Для каждой из трех поцгрупп будут даны доказательства существования указанных выше пределов и вычислены значения этих пределов или найдены границы, в которых они лежат. Этим исследованиям посвящены пп. 4-6 и приложение. 
4. Распределения из класса (А), для которых $\gamma>1$. В этом случае при $n \rightarrow \infty$ имеем $y_{n 1}-y_{n 2} \rightarrow 0$. Положим $\tau=-F^{-1}(\beta /(1-\alpha))$. Тогда

$$
\begin{aligned}
1-\beta & \leqslant I_{1} \leqslant 1-\frac{\beta^{2}}{1-\alpha}, \\
\frac{\alpha \beta}{1-\alpha} & \leqslant I_{2} \leqslant \frac{2 \beta}{1-\alpha}-\frac{\beta^{2}}{1-\alpha}, \\
1-\frac{2 \beta}{1-\alpha} & \leqslant I_{4} \leqslant 1-\frac{\beta}{1-\alpha}, \\
I_{3} & =I_{9}=1, \quad I_{5}=I_{7}=I_{8}=I_{10}=0, \quad I_{6}=\exp (-2 t) .
\end{aligned}
$$

5. Распределения из класса (А), для которых $\gamma=1$. Без нарушения обшности будем считать, что $b=1$. В этом случае при $n \rightarrow \infty$ разность $y_{n 1}-y_{n 2} \rightarrow c=\ln 2$. Величину $\tau$ найдем из уравнения

$$
\exp (-2 t)[F(-\tau)+t F(-c-\tau)]=\beta \text {. }
$$

Тогда

$$
\begin{aligned}
1-\beta & \leqslant I_{1} \leqslant 1-\beta F(-c-\tau), \\
I_{2} & <1-\exp (-2 t)-\beta F(-c-\tau)+\exp (-2 t)[F(-\tau)+2 F(-c-\tau)], \\
I_{3} & >\exp (-2 t)\left((1-F(-c-\tau))^{2}-F(-\tau)\right), \\
I_{4} & >\exp (-2 t)[1-F(-c-\tau)]^{2}, \\
I_{5} & <\exp (-2 t)(1-F(-\tau)) F(-c-\tau), \\
I_{6} & =\exp (-2 t)(1-F(-\tau)), \quad I_{7}<\exp (-t)(F(-\tau)-F(-c-\tau))^{2}, \\
I_{8} & <\exp (-t) F(-\tau)-\exp (-2 t)(F(-\tau)-F(-c-\tau)), \\
I_{9} & >1-\exp (-2 t) F(-c-\tau)(1+F(-\tau)-F(-c-\tau)) \\
& -\exp (-t)(F(-\tau)-F(-c-\tau))^{2}, \\
I_{10} & <1-\exp (-2 t)(1-F(-c-\tau)) .
\end{aligned}
$$

6. Распределения из класса (А), для которых $0<\gamma<1$, и все распределения из класса (В). В этом случае при $n \rightarrow \infty$ разность $y_{n 1}-y_{n 2} \rightarrow \infty$, величина $\tau$ определяется из уравнения

$$
\exp (-2 t) F(-\tau)=\beta \text {. }
$$

Имеем

$$
\begin{aligned}
I_{1} & \geqslant 1-\beta, \\
1-\exp (-t) & \leqslant I_{2} \leqslant 1-\exp (-t)+\beta, \quad I_{3}=1-\beta \exp (-t), \quad I_{4}=\exp (-t), \\
I_{5} & =I_{8}=I_{10}=0, \quad I_{6}=\exp (-2 t)(1-F(-c-\tau)), \\
I_{7} & =\exp (-t) F^{2}(-\tau), \quad I_{9}=\exp (-t)(1-F(-\tau))^{2} .
\end{aligned}
$$


7. Приложение. Док а з а т е л с т в о ле м мы 1.1. Для любого $x>0$ имеем

$$
\begin{aligned}
\mathbf{P} & \left\{\frac{|y|}{\left|Y_{(n-m)}-y\right| n^{\Delta}} \geqslant x\right\}=\mathbf{P}\left\{\left|Y_{(n-m)}-y\right| \leqslant \frac{|y|}{x n^{\Delta}}\right\} \\
= & \mathbf{P}\left\{\sum_{i=1}^{n} \mathbb{I}\left\{Y_{i} \geqslant y+\frac{|y|}{x n^{\Delta}}\right\} \leqslant m\right\}-\mathbf{P}\left\{\sum_{i=1}^{n} \mathbb{I}\left\{Y_{i} \geqslant y-\frac{|y|}{x n^{\Delta}}\right\} \leqslant m\right\} \\
= & \sum_{j=0}^{m} C_{n}^{j}\left(1-F\left(y+\frac{|y|}{x n^{\Delta}}\right)\right)^{j} F^{n-j}\left(y+\frac{|y|}{x n^{\Delta}}\right) \\
& -\sum_{j=0}^{m} C_{n}^{j}\left(1-F\left(y-\frac{|y|}{x n^{\Delta}}\right)\right)^{j} F^{n-j}\left(y-\frac{|y|}{x n^{\Delta}}\right) \rightarrow 0
\end{aligned}
$$

при $n \rightarrow \infty$ в силу соотношения (2), поэтому при $n \rightarrow \infty$ и любом целом фиксированном $m \geqslant 0$

$$
\frac{|y|}{\left|Y_{(n-m)}-y\right| n^{\Delta}} \stackrel{\mathbf{P}}{\longrightarrow} 0 .
$$

Аналогичным образом, в силу (3), при $n \rightarrow \infty$ и любом целом фиксированном $m \geqslant 0$

$$
\frac{1}{\left|Y_{(n-m)}-y\right| n^{\Delta}} \stackrel{\mathbf{P}}{\longrightarrow} 0
$$

Далее, используя представление $n^{\varepsilon}\left(\hat{\theta}_{1}-\theta_{1}\right)=\xi_{n}, n^{\delta}\left(\hat{\theta}_{2}-\theta_{2}\right)=\eta_{n}$, получим следуюшую щепочку соотношений:

$$
\begin{aligned}
& Z_{(n-m)}-y=\frac{X_{(n-m)}-\theta_{1}-\xi_{n} n^{-\varepsilon}}{\theta_{2}+\eta_{n} n^{-\delta}}-y=\left[Y_{(n-m)}-y\right] \\
& \times\left[1+\frac{y}{Y_{(n-m)}-y} O_{p}\left(n^{-\delta}\right)+\frac{1}{Y_{(n-m)}-y} O_{p}\left(n^{-\varepsilon}\right)+O_{p}\left(n^{-\delta}\right)\right],
\end{aligned}
$$

что и требовалось.

Д о к а з а т е л ь с т в о с л е д с т в и я 1.1. Предварительно заметим, что условия (2) и (16) эквивалентны. Действительно, так как

$$
\begin{aligned}
& F\left(y-\frac{|y|}{x n^{\Delta}}\right) \leqslant F(y) \leqslant F\left(y+\frac{|y|}{x n^{\Delta}}\right), \\
& F^{n}\left(y+\frac{|y|}{x n^{\Delta}}\right)-F^{n}\left(y-\frac{|y|}{x n^{\Delta}}\right) \longrightarrow 0, \\
& n F^{n-1}\left(y+\frac{|y|}{x n^{\Delta}}\right)\left(1-F\left(y+\frac{|y|}{x n^{\Delta}}\right)\right) \\
& \quad-n F^{n-1}\left(y-\frac{|y|}{x n^{\Delta}}\right)\left(1-F\left(y-\frac{|y|}{x n^{\Delta}}\right)\right) \longrightarrow 0,
\end{aligned}
$$


и выполняется условие (1), то из (16) следует (2). Аналогичным образом, из условия (17) следует (3).

Для доказательства п. 1) следствия рассмотрим три возможных случая (см. [2]).

1) Предельное распределение типа I для максимума. Тогда $b_{n}=0$, $y_{n}(t) \rightarrow \infty, y_{n}(t) / a_{n} \rightarrow c \neq 0, Y_{(n)} / a_{n}$ при $n \rightarrow \infty$ имеет предельное невырожденное распределение. Имеем

$$
\frac{\left|Y_{(n)}-c a_{n}\right| n^{\Delta}}{c a_{n}}=\left|\frac{Y_{(n)}}{c a_{n}}-1\right| n^{\Delta} \stackrel{\mathbf{P}}{\rightarrow} \infty
$$

2) Предельное распределение типа II для максимума. Тогда либо а) $b_{n}=0, y_{n}(t) \rightarrow 0, a_{n}=-y_{n}(1), y_{n}(t) / y_{n}(1) \rightarrow c$, и, значит,

$$
\frac{\left|Y_{(n)}+c a_{n}\right| n^{\Delta}}{\left|c a_{n}\right|}=\left|\frac{Y_{(n)}}{c a_{n}}+1\right| n^{\Delta} \longrightarrow \infty
$$

либо

б) $b_{n}=c_{1} \neq 0, y_{n}(t) \rightarrow c_{1}$, и, значит,

$$
\frac{\left|Y_{(n)}-c_{1}\right| n^{\Delta}}{\left|c_{1}\right|}=\frac{\left|Y_{(n)}-b_{n}\right| n^{\Delta}}{\left|b_{n}\right|}=\frac{\left|y_{(n)}-b_{n}\right|}{a_{n}} \frac{a_{n} n^{\Delta}}{\left|b_{n}\right|} \longrightarrow \infty .
$$

3) Предельное распределение типа III для максимума. Тогда $b_{n} \rightarrow \infty, y_{n}(t) / b_{n} \rightarrow c \neq 0$ и

$$
\begin{aligned}
\frac{\left|Y_{(n)}-c b_{n}\right| n^{\Delta}}{c b_{n}} & =\frac{\left|Y_{(n)}-b_{n}+b_{n}-c b_{n}\right|}{c a_{n}} \frac{a_{n} n^{\Delta}}{b_{n}} \\
& =\left|\frac{Y_{(n)}-b_{n}}{c a_{n}}+\frac{(1-c) b_{n}}{c a_{n}}\right| \frac{a_{n} n^{\Delta}}{b_{n}} \rightarrow \infty
\end{aligned}
$$

Пункт 2) следствия доказывается аналогичным образом.

Д о к а 3 а т е л ь с т в о т е о.р е м ы 1.1 основано на том факте, что при каждом фиксированном $k \geqslant 0$

$$
\lim _{n \rightarrow \infty} \mathbf{P}\left\{Y_{(n)}<y_{n 1}, Y_{(n-1)}<y_{n 2}, \ldots, Y_{(n-k+1)}<y_{n k}\right\}=\sum_{j=0}^{k-1} p_{k j}
$$

(см., например, [10] или монографию [3, ч. 1, гл. 2, § 2.3]). Утверждение теоремы следует из соотношений (4) и (7).

Д о к а з а т л ь с т о л е м м 2.1 основано на представлении

$$
\left\{Y_{(k)}<x\right\}=\left\{\sum_{j=1}^{n} \mathbb{I}\left\{Y_{j} \geqslant x\right\} \leqslant n-k\right\}
$$


из которого следует, что

$$
\begin{aligned}
I= & \mathbf{P}\left\{\bigcap_{i=1}^{2}\left(\sqrt{n}\left(Y_{\left(n_{i}\right)}-\zeta_{i}\right)<x_{i}\right)\right\}=\mathbf{P}\left\{\bigcap _ { i = 1 } ^ { 2 } \left(\sum_{j=1}^{n-2} \mathbb{I}\left\{Y_{m_{j}} \geqslant \zeta_{i}+\frac{x_{i}}{\sqrt{n}}\right\}\right.\right. \\
& \left.\left.+\mathbb{I}\left\{Y_{c_{1}} \geqslant \zeta_{i}+\frac{x_{i}}{\sqrt{n}}\right\}+\mathbb{I}\left\{Y_{c_{2}} \geqslant \zeta_{i}+\frac{x_{i}}{\sqrt{n}}\right\} \leqslant n-\left[n \lambda_{i}\right]+1\right)\right\} .
\end{aligned}
$$

Tak как

$$
\mathbb{I}\left\{Y_{c} \geqslant \zeta_{i}+\frac{x_{i}}{\sqrt{n}}\right\}=\mathbb{I}\left\{Y_{c}-y_{1}-\tau \geqslant \zeta_{i}+\frac{x_{i}}{\sqrt{n}}-y_{1}-\tau\right\}=1+o_{p}(1)
$$

при $n \rightarrow \infty$ и справедливости гипотезы $H_{1}$, то

$$
I=\mathbf{P}\left\{\bigcap_{i=1}^{2}\left(\sum_{j=1}^{n-2} \mathbb{I}\left\{Y_{m_{j}} \geqslant \zeta_{i}+\frac{x_{i}}{\sqrt{n}}\right\}+o_{p}(1) \leqslant n-\left[n \lambda_{i}\right]-1\right)\right\}
$$

величины $Y_{m_{1}}, \ldots, Y_{m_{n-2}}$ независимы и одинаково распределены с плотностью $f(x)$,

$$
\begin{aligned}
& \mathbf{E} \mathbb{I}\left\{Y_{m_{j}} \geqslant \zeta_{i}+\frac{x_{i}}{\sqrt{n}}\right\}=1-F\left(\zeta_{i}+\frac{x_{i}}{\sqrt{n}}\right) \\
& =\left(1-\lambda_{i}\right)-f\left(\zeta_{i}\right) \frac{x_{i}}{\sqrt{n}}+o\left(n^{-1 / 2}\right), \\
& \operatorname{cov}\left(\mathbb{I}\left\{Y_{m_{1}} \geqslant \zeta_{1}+\frac{x_{1}}{\sqrt{n}}\right\}, \mathbb{I}\left\{Y_{m_{1}} \geqslant \zeta_{2}+\frac{x_{2}}{\sqrt{n}}\right\}\right) \\
& =\lambda_{1}\left(1-\lambda_{2}\right)+o(1), \quad \zeta_{1} \leqslant \zeta_{2} .
\end{aligned}
$$

Таким образом, при $n \rightarrow \infty$ и при справедливости гипотезы $H_{1}$

$$
\begin{gathered}
I=\mathbf{P}\left\{\bigcap _ { i = 1 } ^ { 2 } \left(\frac{\sum_{j=1}^{n-2}\left(\mathbb{I}\left\{Y_{m_{j}} \geqslant \zeta_{i}+x_{i} / \sqrt{n}\right\}-\left(1-F\left(\zeta_{i}+x_{i} / \sqrt{n}\right)\right)\right)}{\sqrt{n} \sqrt{\lambda_{i}\left(1-\lambda_{i}\right)+o(1)}}\right.\right. \\
\left.\left.\leqslant \frac{f\left(\zeta_{i}\right) x_{i}+o_{p}(1)}{\sqrt{\lambda_{i}\left(1-\lambda_{i}\right)+o(1)}}\right)\right\} .
\end{gathered}
$$

Применяя многомерную пентральную предельную теорему к последовательности независимых одинаково распределенных двумерных векторов

$$
\left(\mathbb{I}\left\{Y_{m_{j}} \geqslant \zeta_{1}+\frac{x_{1}}{\sqrt{n}}\right\}, \mathbb{I}\left\{Y_{m_{j}} \geqslant \zeta_{2}+\frac{x_{2}}{\sqrt{n}}\right\}\right), \quad j=1,2, \ldots,
$$

получим, что

$$
\lim _{n \rightarrow \infty} I=\Phi\left(\frac{f\left(\zeta_{1}\right) x_{1}}{\sqrt{\lambda_{1}\left(1-\lambda_{1}\right)}}, \frac{f\left(\zeta_{2}\right) x_{2}}{\sqrt{\lambda_{2}\left(1-\lambda_{2}\right)}}\right)
$$


где $\Phi(\cdot, \cdot)$ означает функцию двумерного нормального распределения с нулевым вектором математических ожиданий и ковариационной матрицей $\Lambda$, что и требовалось.

Дока за т ельст во ле м мы 2.2. Так как мы положили $k=2$, то ограничимся случаями $m=0$ и $m=1$. Напомним, что в п. 1 мы показали, что для классов (А) и (В) выполняются условия (5) и (6), а следовательно, и условия (2) и (3).

Сначала будем считать, что среди $X_{1}, \ldots, X_{n}$ существуют ровно два н.з.в., для которых $\theta_{1 i}=\theta_{2}\left(y_{n 1}+\tau\right)+\theta_{1}$; для остальных $n-2$ наблюдений $\theta_{1 i}=\theta_{1}$. Для любого $x>0$ имеем

$$
\begin{aligned}
& \mathbf{P}\left\{\frac{y}{\left|Y_{(n-1)}-y\right| n^{\Delta}} \geqslant x\right\}=\mathbf{P}\left\{\left|Y_{(n-1)}-y\right| \leqslant \frac{y}{x n^{\Delta}}\right\} \\
& =\mathbf{P}\left\{\sum_{i=1}^{n} \mathbb{I}\left\{Y_{i} \geqslant y+\frac{y}{x n^{\Delta}}\right\} \leqslant 1\right\}-\mathbf{P}\left\{\sum_{i=1}^{n} \mathbb{I}\left\{Y_{i} \geqslant y-\frac{y}{x n^{\Delta}}\right\} \leqslant 1\right\} \\
& =F^{n-2}\left(y+\frac{y}{x n^{\Delta}}\right) F^{2}\left(\frac{y}{x n^{\Delta}}-\tau\right) \\
& +2\left(1-F\left(\frac{y}{x n^{\Delta}}-\tau\right)\right) F\left(\frac{y}{x n^{\Delta}}-\tau\right) F^{n-2}\left(y+\frac{y}{x n^{\Delta}}\right) \\
& +(n-2)\left(1-F\left(y+\frac{y}{x n^{\Delta}}\right)\right) F^{n-3}\left(y+\frac{y}{x n^{\Delta}}\right) F^{2}\left(\frac{y}{x n^{\Delta}}-\tau\right) \\
& -F^{n-2}\left(y-\frac{y}{x n^{\Delta}}\right) F^{2}\left(-\frac{y}{x n^{\Delta}}-\tau\right) \\
& -2\left(1-F\left(-\frac{y}{x n^{\Delta}}-\tau\right)\right) F\left(-\frac{y}{x n^{\Delta}}-\tau\right) F^{n-2}\left(y-\frac{y}{x n^{\Delta}}\right) \\
& -(n-2)\left(1-F\left(y-\frac{y}{x n^{\Delta}}\right)\right) F^{n-3}\left(y-\frac{y}{x n^{\Delta}}\right) F^{2}\left(-\frac{y}{x n^{\Delta}}-\tau\right) \\
& \sim \exp (-t) F^{2}(-\tau)+2(1-F(-\tau)) F(-\tau) \exp (-t)+t \exp (-t) F^{2}(-\tau) \\
& -\exp (-t) F^{2}(-\tau)-2(1-F(-\tau)) F(-\tau) \exp (-t) \\
& -t \exp (-t) F^{2}(-\tau)=0 \text {. }
\end{aligned}
$$

Здесь мы использовали условие $(2)$, соотношения $(8),(9)$ и условие $\Delta \gamma>$ 1 для класса (В). Отсюда следует, что при альтернативе $H_{1}=H_{11} \cup H_{12}$ и при $n \rightarrow \infty$

$$
\frac{y}{\left|Y_{(n-1)}-y\right| n^{\Delta}} \stackrel{\mathbf{P}}{\longrightarrow} 0
$$

Аналогичным образом доказывается, что при альтернативе $H_{1}=H_{11} \cup$ 
$H_{12}$ и $n \rightarrow \infty$

$$
\frac{1}{\left|Y_{(n-i)}-y\right| n^{\Delta}} \stackrel{\mathbf{P}}{\longrightarrow} 0, \quad i=0,1, \quad \frac{y}{\left.\left|Y_{(n)}-y\right|\right|^{\Delta}} \stackrel{\mathbf{P}}{\longrightarrow} 0 .
$$

Случай, когда существует ровно одно н.з.в., для которого $\theta_{1 i}=\theta_{2}\left(y_{n 1}+\right.$ $\tau)+\theta_{1}$, а для остальных $n-1$ наблюдений $\theta_{1 i}=\theta_{1}$, рассматривается аналогичным образом. Дальнейшие рассуждения полностью повторяют рассуждения из доказательства леммы 1.1, следуюшие после соотношения (17). Лемма 2.2 доказана.

Прежде чем доказывать соотношения из пII. 4-6, сделаем некоторые предварительные замечания, касаюшиеся поведения разности $y_{n 1}-y_{n 2}$ и величины $F^{n}\left(y_{n 1}+\delta\right)$ при $n \rightarrow \infty$.

Для класса (А) из соотношения (8) следует, что

$$
y_{n 1}-y_{n 2}=C\left(\left(\ln \frac{n}{t}\right)^{1 / \gamma}-\left(\ln \frac{n}{2 t}\right)^{1 / \gamma}\right) \sim C\left(\ln \frac{n}{t}\right)^{1 / \gamma-1} \frac{\ln 2}{\gamma}
$$

поэтому для класса (А) при $n \rightarrow \infty$ имеем

$$
y_{n 1}-y_{n 2} \rightarrow \begin{cases}0, & \gamma>1, \\ C \ln 2, & \gamma=1, \\ \infty, & 0<\gamma<1 .\end{cases}
$$

Для класса (В) из соотношения (9) следует, что при $n \rightarrow \infty$ разность $y_{n 1}-y_{n 2} \rightarrow \infty$.

Далее, для распределений из класса (А) при $n \rightarrow \infty$ имеем: при $\gamma>1$

$$
\begin{array}{ll}
F^{n}(y+\delta) \longrightarrow 0 & \text { при } \delta<0, \\
F^{n}(y+\delta) \longrightarrow 1 & \text { при } \delta>0
\end{array}
$$

при $\gamma=1$

$$
F^{n}(y+\delta) \longrightarrow \exp (-t \exp (-b \delta)) \quad \text { при любом } \delta ;
$$

при $0<\gamma<1$

$$
F^{n}(y+\delta) \longrightarrow \exp (-t) \quad \text { при любом } \delta .
$$

Для распределений из класса (В) при $n \rightarrow \infty$ имеем

$$
F^{n}(y+\delta) \longrightarrow \exp (-t) \quad \text { при любом } \delta .
$$

Д ок аз а т е л в с т в о соотношений из п. 4. Совместная ф.р. $Y_{(n-1)}$ и $Y_{(n)}$ при $x \leqslant y$, когда два наблюдения среди $Y_{1}, \ldots, Y_{n}$ сдвинуты вправо на величины $\eta_{11}$ и $\eta_{12}$, имеет вид

$$
\mathbf{P}\left\{Y_{(n-1)}<x, Y_{(n)}<y\right\}=F^{n-2}(x) F\left(x-\eta_{11}\right) F\left(x-\eta_{12}\right)
$$




$$
\begin{aligned}
& +F^{n-2}(x) F\left(x-\eta_{11}\right)\left(\left(F\left(y-\eta_{12}\right)-F\left(x-\eta_{12}\right)\right)\right. \\
& +F^{n-2}(x) F\left(x-\eta_{12}\right) \\
& \times\left(F\left(y-\eta_{11}\right)-F\left(x-\eta_{11}\right)\right)+(n-2) F^{n-3}(x) F\left(x-\eta_{11}\right) \\
& \times F\left(x-\eta_{12}\right)(F(y)-F(x)) .
\end{aligned}
$$

Отсюда следует, что

$$
\lim \mathbf{P}_{12}\left\{D_{0}\right\}=(1-\alpha) F^{2}(-\tau), \quad \lim \mathbf{P}_{11}\left\{D_{0}\right\}=(1-\alpha) F(-\tau) .
$$

Taк как $\min \left(\mathbf{P}_{11}\left\{D_{0}\right\}, \mathbf{P}_{12}\left\{D_{0}\right\}\right) \leqslant \mathbf{P}_{1}\left\{D_{0}\right\} \leqslant \max \left(\mathbf{P}_{11}\left\{D_{0}\right\}, \mathbf{P}_{12}\left\{D_{0}\right\}\right)$, то отсюда следует утверждение (10). Далее,

$$
\begin{gathered}
\lim \mathbf{P}_{12}\left\{D_{0} E\right\}=\lim \mathbf{P}\left\{\bigcap_{i=1}^{n-2}\left(Y_{m_{i}}<\min \left(Y_{c_{1}}, Y_{c_{2}}\right)<y_{n 2}\right)\right. \\
\left.\bigcap\left(\max \left(Y_{c_{1}}, Y_{c_{2}}\right)<y_{n 1}\right)\right\} \\
=2 \lim \int_{-\infty}^{y_{n 2}-y_{n 1}-\tau}[F(-\tau)-F(u)] F^{n-2}\left(u+y_{n 1}+\tau\right) f(u) d u
\end{gathered}
$$

Из соотношения (18) следует, что $\lim \mathrm{P}_{12}\left\{D_{0} E\right\}=0$.

Аналогичным образом,

$$
\lim \mathbf{P}_{11}\left\{D_{0} E\right\}=\lim \int_{-\infty}^{-\tau} F^{n-1}\left(u+y_{n 1}+\tau\right) f(u) d u=0
$$

Следовательно, $\lim \mathbf{P}_{1}\left\{D_{0} E\right\}=0$.

Далте,

$$
\begin{aligned}
\lim \mathbf{P}_{12}\{E\} & =2 \lim \int_{-\infty}^{\infty}(1-F(u)) F^{n-2}\left(u+y_{n 1}+\tau\right) f(u) d u \\
& =2 \lim \int_{-\tau}^{\infty}[1-F(u)] f(u) d u=\left[1-\frac{\beta}{1-\alpha}\right]^{2}
\end{aligned}
$$

Аналотичным образом,

$\lim \mathbf{P}_{11}\{E\}=\lim \int_{-\infty}^{\infty} F^{n-1}\left(x+y_{n 1}+\tau\right) f(x) d x=1-F(-\tau)=1-\frac{\beta}{1-\alpha}$

Таким образом, $(1-\beta /(1-\alpha))^{2} \leqslant \lim \mathbf{P}_{1}\{E\} \leqslant 1-\beta /(1-\alpha)$. Так как

$$
\mathbf{P}_{1}\left\{D_{1} E\right\}=\mathbf{P}_{1}\{E\}-\mathbf{P}_{1}\left\{D_{0} E\right\}
$$

то отсюда следует, что

$$
\lim \mathbf{P}_{1}\left\{D_{1} E\right\}=\lim \mathbf{P}_{1}\{E\}
$$


И

$$
\left(1-\frac{\beta}{1-\alpha}\right)^{2} \leqslant \lim \mathbf{P}_{1}\left\{D_{1} E\right\} \leqslant 1-\frac{\beta}{1-\alpha}
$$

что доказывает соотношение (11).

Из равенства

$$
\mathbf{P}_{1}\left\{D_{1} \mid E\right\} \mathbf{P}_{1}\{E\}=\mathbf{P}_{1}\left\{D_{1} E\right\}
$$

и из соотношения (26) следует, что $I_{3}=1$. Остальные соотношения из п. 4 получаются аналогичным образом.

Д о ка за т е л ь с т в о соотношений из п. 5. Из соотношений (19) и (22) следует, что

$$
\begin{aligned}
& \lim \mathbf{P}_{12}\left\{D_{0}\right\}=\exp (-2 t) F(-c-\tau)[2 F(-\tau)-F(-c-\tau)+t F(-c-\tau)] \\
& \lim \mathbf{P}_{11}\left\{D_{0}\right\}=\exp (-2 t)[F(-\tau)+t F(-c-\tau)]
\end{aligned}
$$

откуда получаем утверждение (12). Из соотнопения (23) следует, что

$$
\lim \mathbf{P}_{12}\left\{D_{0} E\right\} \leqslant \exp (-2 t) F^{2}(-c-\tau) \text {. }
$$

Аналогичным образом, $\lim \mathbf{P}_{11}\left\{D_{0} E\right\} \leqslant \exp (-2 t) F(-\tau)$. Таким образом, $\lim \mathbf{P}_{1}\left\{D_{0} E\right\} \leqslant \exp (-2 t) F(-\tau)$. Далее, учитывая соотношение (24), получим

$$
\begin{aligned}
\lim \mathbf{P}_{12}\{E\} & >2 \lim \int_{-c-\tau}^{\infty} F^{n-2}\left(u+y_{n 1}+\tau\right)[1-F(u)] f(u) d u \\
& >2 \exp (-2 t) \int_{-c-\tau}^{\infty}[1-F(u)] f(u) d u \\
& =\exp (-2 t)[1-F(-c-\tau)]^{2}
\end{aligned}
$$

Аналогичным образом,

$$
\lim \mathbf{P}_{11}\{E\}>\exp (-2 t) \int_{-c-\tau}^{\infty} f(u) d u=\exp (-2 t)[1-F(-c-\tau)]
$$

Отсюда слгедует, что

$$
\lim \mathbf{P}_{1}\{E\}>\exp (-2 t)[1-F(-c-\tau)]^{2} .
$$

Используя соотношение (25), получим

$$
\lim \mathbf{P}_{1}\left\{D_{1} E\right\}>\exp (-2 t)\left[(1-F(-c-\tau))^{2}-F(-\tau)\right]
$$

откуда следует соотношение (13).

Соотношение (14) следует из соотношений (27) и (28). Остальные соотношения п. 5 получаются аналогичным образом. 
Д о к а 3 а т е л ь с т в о соотношений из п. 6 основано на использовании соотношений (20) и (21). Методами, аналогичными тем, которые были использованы при доказательстве соотношений из п. 4, получим, что

$$
\lim \mathbf{P}_{12}\left\{D_{0}\right\}=0, \quad \lim \mathbf{P}_{11}\left\{D_{0}\right\}=\exp (-2 t) F(-\tau)=\beta
$$

поэтому $\lim \mathbf{P}_{1}\left\{D_{0}\right\} \leqslant \beta$, откуда следует (15). Остальные соотношения п. 6 получаются аналогичным образом.

\section{СПИСОК ЛИТЕРАТУРЫ}

1. Большев Л. Н. Обнаружение грубых ошибок в результатах наблюдений. - В сб.: Международная летняя школа по теории вероятностей и математической статистике. Варна, 1974, с. 8-41.

2. Галамбои Я. Асимптотическая теория экстремальных порядковых статистик. M.: Наука, 1984.

3. Лидбеттер $M$., Ротсен $X$., Лиядерея Г. Эхстремумы случайных последовательностей и процессов. М.: Мир, 1989.

4. Пагурова В. И., Нестерова С. А. О слабой сходимости считающих процессов в присутствии мешающих параметров. - Теория вероятн. и ее примен., 1991, т. 36 , в. 1 , с. $176-183$.

5. Пагурова В. И., Чижихова И. Л. Критерии обнаружения выбросов, использующие робастные оценки мешающих параметров. - Теория вероятн. и ее примен., 1995, т. 40 , в. 2 , с. $445-452$.

6. Пагурова В. И., ПІведов А. В. Об асимптотическом распределении максимума стационарной гауссовской последовательности. - Вестник МГУ, сер. 15, 1995, B. 4, c. $33-43$.

7. Barnett V., Lewis T. Outliers in Statistical Data. New York: Wiley, 1984.

8. Хампель Ф., Рончетmи Э., Рауссеу П., IIтаэль В. Робастность в статистике. Подход на основе функций влияния. М.: Мир, 1989.

9. Хьюбер Дж. П. Робастность в статистике. М.: Мир, 1984, 304 с.

10. Убайдуллаева $M$. Об отбраковке резко выделяющихся наблюдений. - Тёория вероятн. и ее примен., 1974, т. XIX, в. 4, с. 864-868. 